\title{
ASPECTOS CLINICOEPIDEMIOLÓGICOS DE LAS PATOLOGÍAS AMBULATORIAS DEL OÍDO FRECUENTES EN ADULTOS
}

\section{Outpatient Clinical-Epidemiological Aspects of Diseases of the Ear Common in Adults}

\author{
Fernanda PINEDA-GEA \\ Universidad Católica Redemptoris Mater Facultad de Ciencias Médicas. Managua. Nicaragua. \\ Correspondencia:ferpgea@gmail.com
}

Fecha de recepción: 11 de mayo de 2020

Fecha de aceptación: 14 de junio de 2020

Fecha de publicación: 16 de junio de 2020

Fecha de publicación del fascículo: 1 de marzo de 2021

Conflicto de intereses: Los autores declaran no tener conflictos de intereses

Imágenes: Los autores declaran haber obtenido las imágenes con el permiso de los pacientes

Política de derechos y autoarchivo: se permite el autoarchivo de la versión post-print (SHERPA/RoMEO)

Licencia CC BY-NC-ND. Licencia Creative Commons Atribución-NoComercial-SinDerivar 4.0 Internacional

Universidad de Salamanca. Su comercialización está sujeta al permiso del editor

RESUMEN: Introducción y objetivo: La patología otorrinolaringología tiene una alta incidencia en afecciones agudas como crónicas en la consulta externa de ORL, pese a esto, no existen datos estadísticos exactos, confiables y estandarizados sobre el perfil epidemiológico de las patologías otológicas ambulatorias en nuestro medio. El objetivo de este estudio fue describir las características clínicas, epidemiológicas, de tratamiento y seguimiento propios de las tres patologías otológicas ambulatorias diagnosticadas frecuentemente en la consulta otorrinolaringológica en nuestra región. Método: Se realizó un estudio observacional retrospectivo, en 312 pacientes con patologías otológicas, las variables estudiadas fueron procesadas a través del programa estadístico IBM-SPSS ${ }^{\circledast}$, aplicando una técnica estadística descriptiva. Resultados: Los tres diagnósticos más frecuentes en consulta externa fueron: otitis media crónica en el $60.6 \%$, predomino en mujeres con el $44 \% ; 12.8 \%$ presbiacusia, en la cual no hubo distinción de sexo y $12.17 \%$ otomicosis afectando a mujeres en el $65.7 \%$. Tuvieron una mayor prevalencia en mayores de 51 años, procedentes del área urbana, sin ninguna formación profesional, manifestándose como hipoacusia, otorrea, otalgia y prurito ótico, referidas de manera aislada o en combinación. Se evidenció que la otomicroscopía, impedanciometría y audiometría tonal liminal fueron las principales técnicas otológicas realizadas para el diagnóstico. El tratamiento farmacológico en procesos infecciosos fue ciprofloxacino oral y ótico, fluconazol oral asociado 


\section{ASPECTOS CLINICOEPIDEMIOLÓGICOS DE LAS PATOLOGÍAS AMBULATORIAS DEL OÍDO FRECUENTES EN ADULTOS PINEDA-GEA F}

clotrimazol o clioquinol ótico, se observó la prescripción de loratadina en el $91.5 \%$ de los pacientes con OMC, $40 \%$ en los pacientes con presbiacusia y un $73.6 \%$ en pacientes con otomicosis. Las principales complicaciones clínicas se encontraron en pacientes con OMC tras una segunda evaluación, las cuales fueron parálisis facial $2.1 \%$ y colesteatoma adquirido asociado a otomastoiditis crónica en el $0.96 \%$.Discusión: La prevalencia y afectación en cuanto al sexo por OMC, presbiacusia y otomicosis discrepa con lo obtenido en otras investigaciones aunque su prevalencia con respecto a la edad se asemeja a lo reportado por otros investigadores Las técnicas complementarias de diagnóstico y el tratamiento se alinean a lo descrito en la literatura, pese a esto, se identificó la prescripción de antihistamínico las cual no fue fundamentada en el expediente clínico, se observó que el uso de antibióticos tópico en combinación por vía oral no fue del todo eficaz para evitar reagudizaciones. Conclusiones: Los diagnósticos más frecuentes fueron OMC, presbiacusia y otomicosis, prevalecieron en mujeres entre la quinta década de vida. El tratamiento diagnóstico y terapéutico de las patologías ambulatorias de etiología infecciosa coincide con la literatura, salvo la prescripción de antihistamínicos la cual fue común en el grupo poblacional, así mismo se identificó que el uso significativo de antibióticos orales, más allá de la terapia tópica, no es del todo eficaz para evitar recidivas y complicaciones.

PALABRAS CLAVE: Epidemiologia y clínica de las patologías ambulatorias del oído; Uso de antihistamínicos; Terapia prologada con antibióticos orales y tópicos, OMC y otomicosis en adultos.

SUMMARY: Introduction and objective: Otorhinolaryngology pathology has a high incidence in acute and chronic conditions in the ENT outpatient clinic, despite this; there are no accurate, reliable and standardized statistical data on the epidemiological profile of ambulatory otology pathologies in our setting. The objective of this study was to describe the clinical, epidemiological, treatment and follow-up characteristics of the three outpatient otological pathologies frequently diagnosed in the otorhinolaryngological consultation in our region. Method: A retrospective observational study was carried out in 312 patients with otological pathologies; the variables studied were processed through the IBM-SPSS ${ }^{\oplus}$ statistical program, applying a descriptive statistical technique. Results: The three most frequent outpatient diagnoses were: chronic otitis media in $60.6 \%$, predominated in women with $44 \% ; 12.8 \%$ presbycusis, in which there was no sex distinction and $12.17 \%$ otomycosis affecting women in $65.7 \%$. They had a higher prevalence in people older than 51 years, from the urban area, without any professional training, manifesting as hearing loss, otorrhea, otalgia and otic pruritus, referred in isolation or in combination. It was evident that otomicroscopy, impedanciometry and liminal tone audiometry were the main otological techniques performed for the diagnosis. Pharmacological treatment in infectious processes was oral and otic ciprofloxacin, oral fluconazole associated clotrimazole or clioquinol otic, the prescription of loratadine was observed in $91.5 \%$ of patients with OMC, $40 \%$ in patients with presbycusis and $73.6 \%$ in patients with otomycosis. The main clinical complications were found in patients with OMC after a second evaluation, which were facial paralysis $2.1 \%$ and acquired cholesteatoma associated with chronic otomastoiditis in $0.96 \%$. Discussion: The prevalence and involvement in terms of sex due to $\mathrm{OMC}$, presbycusis and otomycosis disagrees with that obtained in other investigations although its prevalence with respect to age is similar to that reported by other researchers. Complementary diagnostic and treatment techniques are aligned with that described in the literature, despite this; the prescription of antihistamine was identified. Which was not based on the clinical record, it was observed that the use of topical antibiotics in combination orally was not entirely effective in avoiding flare-ups. Conclusions: The most frequent diagnoses were OMC, presbycusis and otomicosis they prevailed in women between the fifth decade of life. The diagnostic and therapeutic treatment of ambulatory pathologies of infectious etiology coincides with the literature, except for the prescription of antihistamines, which was common in the population group, and it was also identified that the significant use of oral antibiotics, beyond topical therapy, It is not entirely effective in preventing recurrences and complications.

KEYWORDS: Epidemiology and clinic of ambulatory ear pathologies; Use of antihistamines; Prolonged therapy with oral and topical antibiotics; OMC and otomycosis in adults. 


\section{ASPECTOS CLINICOEPIDEMIOLÓGICOS DE LAS PATOLOGÍAS AMBULATORIAS DEL OÍDO FRECUENTES EN ADULTOS \\ PINEDA-GEA F}

\section{INTRODUCCION}

Las patologías otorrinolaringológicas tienen una alta Incidencia tanto de afecciones agudas como crónicas que representan según Seymour y cols. alrededor del $40 \%$ del total de consultas [1]. Pese a que son una condición clínica común, no existen datos estadísticos exactos, confiables y estandarizados sobre la incidencia y prevalencia del perfil epidemiológico de las patologías otológicas ambulatorias en nuestro medio, con respecto a esto Castaño destaca la escasa información estadística disponible en países latinoamericanos [2]. La mayoría de la literatura médica se centra en el diagnóstico, el tratamiento y las complicaciones de las patologías otológicas pediátrica, y gran parte de nuestra información sobre estas patologías en adultos se extrapola de estudios en niños.

Considerando que los procesos infecciosos agudos y crónicos de oído siguen siendo una incertidumbre en muchos aspectos, conocer el comportamiento clínico y epidemiológico de las tres principales patologías ambulatorias en adultos a través de su sintomatología, estudios audiológicos, nos abrirá las puertas para aclarar muchos aspectos que envuelven esta afecciones y con ello estimar el impacto socioeconómico que genera al sector salud en nuestro medio.

El objetivo de este estudio fue describir las características clínicas, epidemiológicas, de tratamiento y seguimiento propios de las tres patologías otológicas ambulatorias diagnosticadas frecuentemente en la consulta otorrinolaringológica en nuestra región.

\section{MATERIAL Y MÉTODO}

Se efectuó un estudio observacional retrospectivo, durante el periodo comprendido entre el 1 de enero al 31 de diciembre de 2018, el universo estuvo compuesto por 3600 pacientes con patologías ambulatorias del oído que acudieron a consulta externa, la muestra fue de 312 expedientes clínicos, calculada mediante la fórmula estadística para poblaciones finitas [3] y elegida a través de una técnica de muestreo probabilístico sistemático [3]. Se incluyeron expedientes clínicos de pacientes con edades mayores 25 años con diagnostico de patologías otológicas. El procesamiento estadístico de las variables en estudio se realizó con el programa IBM-SPSS $^{\circledast}$ (versión 25.0, 2017), aplicando un análisis descriptivo, expresando en frecuencia y porcentajes los resultados obtenidos.

Esta investigación fue aprobada por el comité de ética de la Universidad Católica Redemptoris Mater (UNICA), el Ministerio Salud de Nicaragua (MINSA)-SILAIS, Managua y dirección médica del Hospital Escuela Antonio Lenín Fonseca donde se llevó a cabo la investigación.

\section{RESULTADOS}

Las características sociodemográficas prevalentes de la población estudiada se observan en la Tabla 1 donde se describen en frecuencia y porcentaje los resultados obtenidos, los cuales se calcularon en base a la muestra $(\mathrm{n}=312)$ del grupo poblacional sometido a estudio, encontrándose que el $55 \%$ tenía una edad comprendida entre los 51-59 años $\mathrm{o} \geq$ de 60 años. El $64.4 \%$ de la población era femenina, predominando sobre la masculina la cual comprendió el $35.6 \%$. El $84.1 \%$ procedía de la zona urbana. La mayoría del grupo poblacional en el $34.1 \%$ eran amas de casa que no tenían ninguna formación profesional seguido del $12.2 \%$ que tenía formación universitaria. Un 49.7 \% no tenía ningún hábito tóxico (tabaquismo o alcoholismo); Hubo un 7.7 \% que si practicaba conjuntamente el consumo del tabaco y alcohol. Las principales comorbilidades identificadas fueron hipertensión arterial en el $19.9 \%$ y diabetes mellitus tipo II con un $11.5 \%$, las cuales se presentaron de manera asilada o conjunta en los pacientes. El principal factor relacionado a la etiología identificado en las patologías ambulatorias del oído en la población fue en un $85.6 \%$ el antecedente de infección otológica recurrente de oído medio y externo. 


\section{ASPECTOS CLINICOEPIDEMIOLÓGICOS DE LAS PATOLOGÍAS AMBULATORIAS DEL OIIDO FRECUENTES EN ADULTOS \\ PINEDA-GEA F}

Las patologías ambulatorias diagnosticadas, independientemente si se asociaron o no a otras patologías del oído y si afectaron de manera unilateral o bilateral a la población estudiada, se observan en orden de frecuencia en la Tabla 2 , evidenciándose que $60.6 \%$ tenía OMC, afectando el $5.25 \%$ a la población (3600) en el año 2018, $12.8 \%$ presento presbiacusia suponiendo el $1.1 \%$ de las patologías ambulatorias diagnosticadas en $2018,12.7 \%$ otomicosis, suponiendo un $1 \%$ de las patologías del oído diagnosticadas en el año 2018, las características sociodemográficas de estas patologías ambulatorias, se describen en la Tabla 2.1; los porcentajes expresados en esta Tabla, se calcularon basados en el número de pacientes que presentaron estas patologías (prevalencia puntual) sin tomar en cuenta la afectación bilateral o unilateral del oído y si se asociaron o no a otras patologías. Cabe mencionar que las patologías asociadas a otitis media crónica (OMC) presbiacusia y otomicosis se describen en la Tabla 3, en la que no se muestran totales ya que un mismo paciente además de la patología ambulatoria mas frecuente presento una o más patologías asociadas, por lo que el porcentaje de presentación de cada patología asociada a la patología ambulatoria más frecuente se calculó basado en la muestra de pacientes que presentaron la patología ambulatoria.

Tabla 1. Características sociodemográficas prevalentes en la población estudiada.

\begin{tabular}{|l|l|l|}
\hline \multicolumn{2}{|l|}{ n } & $\%$ \\
\hline Edad & 88 & 28.2 \\
\hline 51-59 años & 82 & 26.8 \\
\hline$\geq$ de 60 años & 82 \\
\hline Sexo & 201 & 64.4 \\
\hline Mujer & 111 & 35.6 \\
\hline Varón & &
\end{tabular}

\begin{tabular}{|l|l|l|}
\hline \multicolumn{2}{|l|}{ n } & $\%$ \\
\hline Procedencia & 253 & 81.1 \\
\hline Urbana & 59 & 18.9 \\
\hline Rural & \multicolumn{2}{l}{} \\
\hline Formación profesional & 98 & 31.4 \\
\hline Labores domésticas & 35 & 12.2 \\
\hline Universitaria & \multicolumn{2}{|l}{} \\
\hline Hábitos tóxicos & 155 & 49.7 \\
\hline Ninguno & 24 & 7.7 \\
\hline Tabaco y alcohol & 109 & 34.9 \\
\hline Comorbilidades & 62 & 19.9 \\
\hline Ninguna & 36 & 11.5 \\
\hline Hipertensión arterial (HTA) & 7.05 \\
\hline Diabetes Mellitus tipo II (DMII) & 36 & 85.6 \\
\hline HTA y DM II & 22 & 59.3 \\
\hline Factores etiológicos relacionados & 185 & \\
\hline $\begin{array}{l}\text { Infección otológica recurrente de } \\
\text { oído medio y externo }\end{array}$ & 267 & \\
\hline Edad mayor de 51 anos & \multicolumn{2}{|l}{} \\
\hline
\end{tabular}

En cuanto a las manifestaciones clínicas de las patologías del oído diagnosticadas con mayor frecuencia, se evidencio que del total de pacientes con OMC ( $\mathrm{n}=189)$, un $31.5 \%(\mathrm{n}=60)$ presentó otras patologías asociadas y el $68.2 \%(n=129)$ presento exclusivamente OMC; independientemente de su asociación o no con otras patologías, la hipoacusia en 186 pacientes $(98.41 \%)$ fue el principal síntoma referido. La distribución de presentación de las manifestaciones clínicas referidas por los pacientes con OMC se muestra en la Tabla 4. Del total de pacientes diagnosticados con presbiacusia $(n=40)$ asociada o no a otras patologías, el principal síntoma fue en el $100 \%$ de los pacientes hipoacusia, referida como único síntoma o asociada a otros (Tabla 4.1). 


\section{ASPECTOS CLINICOEPIDEMIOLÓGICOS DE LAS PATOLOGÍAS AMBULATORIAS DEL OÍDO FRECUENTES EN ADULTOS \\ PINEDA-GEA F}

Tabla 2. Patologías ambulatorias diagnosticadas en la población estudiada

\begin{tabular}{|c|c|c|}
\hline Patología & $\mathrm{n}$ & $\%$ \\
\hline Otitis Media Crónica (OMC) & 189 & 60.6 \\
\hline Presbiacusia & 40 & 12.8 \\
\hline Otomicosis & 38 & 12.17 \\
\hline Hipoacusia a estudio & 29 & 9.2 \\
\hline Disfunción de la trompa de Eustaquio & 23 & 7.3 \\
\hline Otomastoiditis aguda & 22 & 7.05 \\
\hline Otitis externa no especificada & 14 & 4.4 \\
\hline Síndrome vertiginosos & 6 & 1.9 \\
\hline Eczema ótico & 6 & 1.9 \\
\hline Acúfenos & 6 & 1.9 \\
\hline Fistula preauricular Infectada & 4 & 1.2 \\
\hline $\begin{array}{l}\text { Estenosis del Conducto Auditivo Externo } \\
\text { (CAE) }\end{array}$ & 4 & 1.2 \\
\hline Tapón de cerumen & 3 & 0.96 \\
\hline Otosclerosis & 3 & 0.96 \\
\hline Granuloma del conducto auditivo externo & 3 & 0.96 \\
\hline Atresia del conducto auditivo externo & 2 & 0.64 \\
\hline Otohematoma & 2 & 0.64 \\
\hline Microtia & 2 & 0.64 \\
\hline $\begin{array}{l}\text { Anotia del pabellón auricular por } \\
\text { arrancamiento }\end{array}$ & 1 & 0.3 \\
\hline Neurinoma del acústico & 1 & 0.3 \\
\hline Disyunción de la cadena timpanooscicular & 1 & 0.3 \\
\hline Disyunción temporomandibular & 1 & 0.3 \\
\hline Fistula antro-cutánea & 1 & 0.3 \\
\hline Trauma acústico agudo & 1 & 0.3 \\
\hline Colesteatoma recurrente & 1 & 0.3 \\
\hline Absceso Preauricular & 1 & 0.3 \\
\hline Carcinoma basocelular del pabellón auricular & 1 & 0.3 \\
\hline Síndrome de Ménière & 1 & 0.3 \\
\hline $\begin{array}{l}\text { Osteoma del Conducto Auditivo Externo } \\
\text { (CAE) }\end{array}$ & 1 & 0.3 \\
\hline $\begin{array}{l}\text { Tumoración a estudio del conducto auditivo } \\
\text { externo }\end{array}$ & 1 & 0.3 \\
\hline Anotia & 1 & 0.3 \\
\hline
\end{tabular}

Los pacientes con otomicosis asociada o no a otras patologías, refirieron en el $94.7 \%(n=36)$ prurito ótico como principal síntoma ya fuese como síntoma único o asociado o no a otros (Tabla 4.2). Los porcentajes de las manifestaciones clínicas referidas de manera única o asociada a otros síntomas, de las patologías diagnosticadas con mayor frecuencia, se calcularon en base a el número de pacientes diagnosticados con estas patologías, por lo que en las Tablas correspondientes, se muestra la prevalencia puntual de las manifestaciones clínicas de estos pacientes con respecto a la patología ambulatoria diagnosticada.

Tabla 2.1. Características sociodemográficas de las patologías prevalentes del oído

\begin{tabular}{|c|c|c|c|c|c|c|}
\hline \multirow[b]{2}{*}{ Edad } & \multicolumn{2}{|c|}{ OMC } & \multicolumn{2}{|c|}{ Presbiacusia } & \multicolumn{2}{|c|}{ Otomicosis } \\
\hline & $\mathrm{n}$ & $\%$ & $\mathrm{n}$ & $\%$ & $\mathrm{n}$ & $\%$ \\
\hline 51-59 años & 53 & 17 & 12 & 30 & 9 & $\begin{array}{l}23.6 \\
8\end{array}$ \\
\hline$\geq \operatorname{de} 60$ años & 42 & 13.4 & 27 & 67.5 & 8 & $\begin{array}{l}21.0 \\
5\end{array}$ \\
\hline \multicolumn{7}{|l|}{ Sexo } \\
\hline Mujer & 136 & 44 & 20 & 50 & 25 & 65.7 \\
\hline Varón & 53 & 17 & 20 & 50 & 13 & 34.2 \\
\hline \multicolumn{7}{|l|}{ Procedencia } \\
\hline Rural & 155 & $\begin{array}{l}82.0 \\
1\end{array}$ & 8 & 20 & 31 & 81.5 \\
\hline Urbana & 34 & $\begin{array}{l}17.9 \\
8\end{array}$ & 32 & 80 & 7 & 18.4 \\
\hline \multicolumn{7}{|l|}{ Ocupación } \\
\hline Labores domésticas & 70 & $\begin{array}{l}37.0 \\
3\end{array}$ & 16 & 40 & 12 & 31.5 \\
\hline \multicolumn{7}{|l|}{ Hábito tóxico } \\
\hline Ninguno & 95 & 50.2 & 16 & 40 & 21 & 55.2 \\
\hline Tabaco y Alcohol & 14 & 7.4 & 7 & 17.5 & 2 & 5.2 \\
\hline \multicolumn{7}{|l|}{ Comorbilidad } \\
\hline HTA & 33 & 17.4 & 12 & 30 & 2 & 5.2 \\
\hline DM II & 14 & 7.4 & 4 & 10 & 0 & 0 \\
\hline HTA + DM II & 8 & 4.2 & 8 & 20 & 2 & 5.2 \\
\hline Ninguna & 74 & 23.7 & 6 & 15 & 16 & 42.1 \\
\hline \multicolumn{7}{|l|}{ Factor etiológico } \\
\hline $\begin{array}{l}\text { Infección } \\
\text { recurrente }\end{array}$ & 182 & 96.2 & 6 & 10 & 36 & $\begin{array}{l}94.7 \\
3 \\
\end{array}$ \\
\hline Edad $\geq$ de 51 años & 189 & 100 & 36 & 90 & 21 & 55.2 \\
\hline Prevalencia & 189 & 60.5 & 40 & 12.8 & 38 & 12.1 \\
\hline
\end{tabular}




\section{ASPECTOS CLINICOEPIDEMIOLÓGICOS DE LAS PATOLOGÍAS AMBULATORIAS DEL OÍDO FRECUENTES EN ADULTOS \\ PINEDA-GEA F}

Se evidenció que la otomicroscopía, impedanciometría y audiometría tonal liminal fueron las principales pruebas otológicas realizadas de manera simultánea en el $44.97 \%(\mathrm{n}=85)$ pacientes con OMC, $77.5 \%(\mathrm{n}=31)$ Presbiacusia y $36.86 \%$ $(\mathrm{n}=14)$ pacientes con otomicosis. La Tomografía Axial Computarizada (TAC) de cráneo y oído simple, fue la principal prueba imagenología realizada en este estudio, el $26.39 \%(\mathrm{n}=50)$ en pacientes con OMC, $7.5 \%(\mathrm{n}=3)$ con presbiacusia asociada a OMC y un $21.05 \%(\mathrm{n}=8)$ en pacientes con otomicosis asociada a OMC, las combinaciones de los prueba complementarias se detallan en la Tabla 5.

Tabla.3 Patologías asociadas a las patologías ambulatorias prevalentes en la población.

\begin{tabular}{|c|c|c|c|c|c|c|}
\hline \multirow{2}{*}{$\begin{array}{l}\text { Patologías } \\
\text { otológicas } \\
\text { ambulatorias }\end{array}$} & \multicolumn{2}{|c|}{$\begin{array}{l}\text { OMC } \\
(\mathrm{n}=61)\end{array}$} & \multicolumn{2}{|c|}{\begin{tabular}{|l|}
$\begin{array}{l}\text { Presbiacusia } \\
(\mathrm{n}=18)\end{array}$ \\
\end{tabular}} & \multicolumn{2}{|c|}{$\begin{array}{l}\text { Otomicosis } \\
(\mathrm{n}=26)\end{array}$} \\
\hline & $\mathrm{n}$ & $\%$ & $\mathrm{n}$ & $\%$ & $\mathrm{n}$ & \\
\hline OMC & 0 & 0 & 11 & 61.1 & 22 & 85 \\
\hline Acufeno & 1 & 1.6 & 3 & 16.6 & 0 & 0 \\
\hline Otomicosis & 22 & 36 & 2 & 11.1 & 0 & 0 \\
\hline Presbiacusia & 15 & 24.5 & 0 & 0 & 2 & 7.6 \\
\hline $\begin{array}{l}\text { Disfunción } \\
\text { tubárica }\end{array}$ & 10 & $\begin{array}{l}16.3 \\
9\end{array}$ & 3 & 16.6 & 2 & 7.6 \\
\hline Mastoiditis & 4 & 6.5 & 0 & 0 & 0 & 0 \\
\hline Eczema ótico & 3 & 4.9 & 1 & 5.5 & 2 & 7.6 \\
\hline Estenosis de CAE & 3 & 4.9 & 0 & 0 & 1 & 3.8 \\
\hline $\begin{array}{l}\text { Otitis externa } \\
\text { inespecífica }\end{array}$ & 3 & 4.9 & 0 & 0 & 0 & 0 \\
\hline Microtia grado II & 2 & 3.2 & 0 & 0 & 0 & 0 \\
\hline $\begin{array}{l}\text { Síndrome } \\
\text { vertiginoso }\end{array}$ & 2 & 3.2 & 2 & 11.1 & 2 & 7.6 \\
\hline Tapón de cerumen & 2 & 3.2 & 1 & 5.5 & 0 & 0 \\
\hline Anotia & 1 & 1.6 & 0 & 0 & 0 & 0 \\
\hline $\begin{array}{l}\text { Colesteatoma } \\
\text { recurrente }\end{array}$ & 1 & 1.6 & 0 & 0 & 0 & 0 \\
\hline $\begin{array}{l}\text { Granuloma del } \\
\text { CAE }\end{array}$ & 1 & 1.6 & 0 & 0 & 0 & 0 \\
\hline $\begin{array}{l}\text { Disfunción } \\
\text { temporomandibular }\end{array}$ & 1 & 1.6 & 0 & 0 & 0 & 0 \\
\hline Otoesclerosis & 1 & 1.6 & 0 & 0 & 0 & 0 \\
\hline $\begin{array}{l}\text { Otomastoiditis } \\
\text { crónica no } \\
\text { colestomatosa }\end{array}$ & 0 & 0 & 0 & 0 & 1 & 3.8 \\
\hline
\end{tabular}

Ediciones Universidad de Salamanca / @@@
Los fármacos prescritos en en las patologías más frecuentes, se muestran en la Tabla 6, estos se prescribieron en cada paciente de manera exclusiva o combinado con otros fármacos, por lo que no se describen totales en esta Tabla, los porcentajes se calcularon tomando en cuenta el número de pacientes a los que se prescribió dicho fármaco y presento una de las tres patologías más frecuentes, ya fuese asilada o asociada a otras patologías. Se evidenció que la ciprofloxacina $500 \mathrm{mg}$ vía oral y ótica asociado a corticoide fue el antibiótico de elección en las patologías infecciosas de origen bacteriano, prescribiéndose en el $44.4 \%(n=84)$ pacientes con OMC, $20 \%$ $(n=8)$ en pacientes con presbiacusia y $18.42 \%(n=7)$ en pacientes con otomicosis. El fluconazol 150mg vía oral o asociado antifúngico ótico (clotrimazol ótico o clioquinol asociado a beclometasona) fue la terapia antimicótica de elección en los pacientes con patologías infecciosas de origen micótico, prescribiéndose en el $7.4 \%(n=14)$ en pacientes con OMC y el $60.5 \%(n=23)$ en pacientes con otomicosis, no prescribiéndose en pacientes con presbiacusia. El analgésico de elección fue el ibuprofeno 400mg vía oral, los antihistamínicos de elección fueron loratadina 10mg vía oral prescribiéndose en el $91.5 \%$ OMC, $45 \%$ Presbiacusia y $73.6 \%$ otomicosis, el cual fue prescrito de forma exclusiva o asociada u otros fármacos. En la terapia antivertiginosa se utilizó el dimenhidrinato $50 \mathrm{mg}$ vía oral prescribiéndose en el 0,5\% $(n=1)$ paciente con OMC, $7.5 \%(n=3)$ presbiacusia y un $5.2 \%(\mathrm{n}=2)$ otomicosis.

Tabla 4. Manifestaciones clínicas presentadas por los pacientes con diagnóstico de OMC.

\begin{tabular}{|l|l|c|}
\hline \multicolumn{4}{|l|}{$\begin{array}{l}\text { Manifestaciones clínicas presentadas por los pacientes con } \\
\text { diagnóstico exclusivo de OMC }\end{array}$} & $\mathrm{n}$ & $\%$ \\
\hline Manifestaciones clínicas & 48 & 37,2 \\
\hline Hipoacusia & 37 & 28,7 \\
\hline Hipoacusia, otorrea & 23 & 17,8 \\
\hline Hipoacusia, otalgia y otorrea & 17 & 13,2 \\
\hline Hipoacusia y otalgia & 2 & 1,6 \\
\hline Hipoacusia, otorrea y prurito ótico & 1 & 0,8 \\
\hline Hipoacusia y acúfeno & 1 & 0,8 \\
\hline Prurito ótico y otorrea & $129(68.2)$ \\
\hline Prevalencia puntual & \multicolumn{3}{|l}{} \\
\hline
\end{tabular}

\footnotetext{
Ediciones Universidad de Salamanca/ Go
} 


\section{ASPECTOS CLINICOEPIDEMIOLÓGICOS DE LAS PATOLOGÍAS AMBULATORIAS DEL OÍDO FRECUENTES EN ADULTOS

\begin{tabular}{|l|l|l|}
\hline \multicolumn{3}{|l|}{$\begin{array}{l}\text { Manifestaciones clínicas referidas por los pacientes con } \\
\text { diagnóstico OMC asociada a otras patologías otológicas } \\
\text { ambulatorias }\end{array}$} \\
\hline Hipoacusia, otalgia y otorrea & 12 & 20 \\
\hline Hipoacusia, prurito ótico y otorrea & 11 & 18,3 \\
\hline Hipoacusia y otorrea & 9 & 15 \\
\hline hipoacusia y otalgia & 4 & 6.7 \\
\hline Hipoacusia & 4 & 6.7 \\
\hline Hipoacusia, otalgia y prurito ótico & 3 & 5 \\
\hline Hipoacusia y sensación de taponamiento ótico & 3 & 5 \\
\hline Otalgia y prurito ótico & 3 & 5 \\
\hline Hipoacusia, otalgia y vértigo & 2 & 3.3 \\
\hline Hipoacusia y prurito ótico & 2 & 3.3 \\
\hline $\begin{array}{l}\text { Hipoacusia, otalgia y sensación de } \\
\text { taponamiento ótico }\end{array}$ & 2 & 3.3 \\
\hline Hipoacusia, otalgia y acufenos & 2 & 3.3 \\
\hline Hipoacusia, otalgia, prurito tico y vértigo & 1 & 1.7 \\
\hline Prurito ótico & 1 & 1.7 \\
\hline $\begin{array}{l}\text { Hipoacusia, otalgia, dehiscencia de herida } \\
\text { retroauricular }\end{array}$ & 1 & 1.7 \\
\hline Prevalencia puntual & 60 & 31.7 \\
\hline
\end{tabular}

Tabla 4.2. Manifestaciones clínicas referidas por pacientes con otomicosis.

\begin{tabular}{|l|l|l|}
\hline \multicolumn{3}{|l|}{$\begin{array}{l}\text { Manifestaciones clínicas referidas por pacientes con } \\
\text { diagnostico exclusivo de otomicosis }\end{array}$} \\
\hline Síntomas & $\mathrm{n}$ & $\%$ \\
\hline Prurito ótico & 5 & 41.7 \\
\hline Prurito ótico y otalgia & 3 & 25 \\
\hline Prurito ótico, otalgia e hipoacusia & 3 & 25 \\
\hline Prurito ótico e hipoacusia & 1 & 8.3 \\
\hline Prevalencia & $12(31.5)$ \\
\hline $\begin{array}{l}\text { Manifestaciones clínicas referidas por pacientes con diagnóstico } \\
\text { de otomicosis asociado a otras patologías otológicas ambulatorias }\end{array}$ \\
\hline Síntomas & \multicolumn{1}{|l|}{} \\
\hline Prurito ótico, otorrea e hipoacusia & 10 & 38.5 \\
\hline Prurito ótico y otalgia & 4 & 15.4 \\
\hline Prurito ótico, otalgia e hipoacusia & 4 & 15.4 \\
\hline Prurito ótico e hipoacusia & 2 & 7.7 \\
\hline Prurito ótico & 1 & 3.8 \\
\hline Prurito ótico y vértigo & 1 & 3.8 \\
\hline Otorrea e hipoacusia & 1 & 3.8 \\
\hline Hipoacusia, otalgia y otorrea & 1 & 3.8 \\
\hline Prurito ótico, hipoacusia, otalgia, vértigo & 1 & 3.8 \\
\hline $\begin{array}{l}\text { Hipoacusia, sensación de taponamiento y } \\
\text { prurito ótico }\end{array}$ & 1 & 3.8 \\
\hline Prevalencia & 26 & 68.2 \\
\hline
\end{tabular}

Los principales hallazgos clínicos presentados tras la terminación del tratamiento evidenciadas durante una segunda reevaluación por el grupo poblacional en que se presentó $\mathrm{OMC}$, presbiacusia y otomicosis, fueron: $59.6 \%(\mathrm{n}=186)$ disminución de la capacidad auditiva (hipoacusia), $30.1 \%(\mathrm{n}=94)$ perforación timpánica, $9.9 \%(\mathrm{n}=31)$ fracaso terapéutico debido a infección ótica persistente del oído medio y externo y $4.8 \%(\mathrm{n}=15)$ acúfeno persistente Los hallazgos clínicos y las principales complicaciones clínicas se muestran en el Tabla 7.

Tabla 5. Métodos complementarios utilizados en el diagnósticos de las patologías ambulatorias más frecuentes del oído.

\begin{tabular}{|c|c|c|c|c|c|c|}
\hline \multirow{2}{*}{$\begin{array}{l}\text { Métodos } \\
\text { complementarios }\end{array}$} & \multicolumn{2}{|c|}{$\mathrm{OMC}$} & \multicolumn{2}{|c|}{ Presbiacusia } & \multicolumn{2}{|c|}{ Otomicosis } \\
\hline & $\mathrm{n}$ & $\%$ & $\mathrm{n}$ & $\%$ & $\mathrm{n}$ & $\%$ \\
\hline $\begin{array}{l}\text { Otoscopía, } \\
\text { audiometría } \\
\text { tonal liminal e } \\
\text { impedanciometría }\end{array}$ & 85 & $\begin{array}{l}44.9 \\
7\end{array}$ & 31 & 77.5 & 14 & $\begin{array}{l}36.8 \\
4\end{array}$ \\
\hline Otoscopía directa & 51 & $\begin{array}{l}29.9 \\
8\end{array}$ & 4 & 10 & 14 & $\begin{array}{l}36.8 \\
4\end{array}$ \\
\hline $\begin{array}{l}\text { TAC de cráneo, } \\
\text { otoscopia, } \\
\text { audiometría } \\
\text { tonal liminal e } \\
\text { impedanciometría }\end{array}$ & 48 & $\begin{array}{l}25.3 \\
9\end{array}$ & 3 & 7.5 & 8 & $\begin{array}{l}21.0 \\
5\end{array}$ \\
\hline $\begin{array}{l}\text { Logoaudiometría, } \\
\text { TAC de cráneo, } \\
\text { otoscopia, } \\
\text { audiometría } \\
\text { tonal liminal, } \\
\text { impedanciometría }\end{array}$ & 1 & 0.5 & 0 & 0 & 0 & 0 \\
\hline $\begin{array}{l}\text { Logoaudiometría, } \\
\text { otoscopia, } \\
\text { audiometría } \\
\text { tonal liminal, } \\
\text { impedanciometría }\end{array}$ & 1 & 0.5 & 1 & 2.5 & 0 & 0 \\
\hline $\begin{array}{l}\text { Prueba de } \\
\text { Romberg y } \\
\text { Otoscopia }\end{array}$ & 1 & 0.5 & 0 & 0 & 0 & 0 \\
\hline $\begin{array}{l}\text { Prueba de } \\
\text { Romberg, } \\
\text { otoscopia, } \\
\text { audiometría } \\
\text { tonal liminal, } \\
\text { impedanciometría }\end{array}$ & 1 & 0.5 & 1 & 2.5 & 2 & 5.26 \\
\hline
\end{tabular}




\begin{tabular}{|l|l|l|l|l|l|l|}
\hline \multirow{2}{*}{$\begin{array}{l}\text { Métodos } \\
\text { complementarios }\end{array}$} & \multicolumn{2}{l|}{ OMC } & \multicolumn{2}{l|}{ Presbiacusia } & \multicolumn{2}{l|}{ Otomicosis } \\
\cline { 2 - 7 } & $\mathrm{n}$ & $\%$ & $\mathrm{n}$ & $\%$ & $\mathrm{n}$ & $\%$ \\
\hline $\begin{array}{l}\text { TAC de cráneo, } \\
\text { otoscopia, } \\
\text { resonancia } \\
\text { magnética } \\
\text { de cráneo, } \\
\text { audiometría } \\
\text { tonal liminal, } \\
\text { impedanciometría, } \\
\text { cultivo de } \\
\text { secreción ótica }\end{array}$ & 1 & 0.5 & 0 & 0 & 0 & 0 \\
\hline Prevalencia puntual & $189(100)$ & $40(100)$ & $38(100)$ \\
\hline
\end{tabular}

Estas complicaciones fueron: parálisis facial $1.9 \%(n=6)$, de los cuales un $2.1 \%(n=4)$ de los pacientes con OMC presento esta complicación, cuya severidad según la clasificación de House Brackmann fue Grado IV, la cual se presentó en un paciente intervenido por shwanoma vestibular del oído izquierdo, quien sufrió infección del sitio quirúrgico y fue reintervenido en segunda instancia e ingresado para su manejo. Dos pacientes con OMC presentaron parálisis facial House Brackmann Grado III y uno presento parálisis facial House Brackmann Grado II.

Cabe añadir que estos pacientes presentaron fracaso terapéutico debido a infección ótica persistente del oído medio, perforación timpánica y disminución de la capacidad auditiva (Hipoacusia), así mismo no se evidencio en pacientes con presbiacusia este tipo de complicación; sin en cambio se presentó parálisis facial House Brackmann grado III en un $2.6 \%(\mathrm{n}=1)$ de la otomicosis asociada a OMC, cabe recalcar, este paciente se complicó además con infección ótica persistente del oído externo (Otomicosis persistente) y fue tratado con solución de ácido acético 5 gotas vía ótica cada 8 horas por 7 días, sin ningún tratamiento farmacológico antibiótico o antifúngico previo. El colesteatoma adquirido asociado a otomastoiditis crónica fue la segunda complicación clínica presentada en el $0.96 \%(n=3)$ de los pacientes, presentándose exclusivamente en OMC.

\section{DISCUSIÓN}

En lo referente al perfil epidemiológico del grupo poblacional valorado, se encontró que la mayor parte de esta se ubica entre la quinta y sexta década de vida, lo cual podría estar explicado por la diferencias en las pirámides poblacionales entre otros países y el nuestro. Se observó que en esta distribución de las edades, las patologías a ambulatorias diagnosticadas con mayor frecuencia fueron OMC, presbiacusia y otomicosis.

Ruz y Cols. [4] Describen el predominio de OMC en el $43.9 \%$ de los pacientes, pese a la similitud del predominio de esta patología con nuestro estudio, hay una diferencia marcada en cuanto a la prevalencia puntual la cual fue de $60.5 \%$, suponiendo una prevalencia del $5.25 \%$ durante el año 2018 discrepando con lo reportado por Browing [5] quien menciona una prevalencia en adultos del Reino Unido, del $1.5 \%$ en OMC con proceso infeccioso activo y $2.6 \%$ en OMC sin proceso infeccioso activo. Con respecto a su predomino por sexo, se encontró una prevalencia del $44 \%$ en el sexo femenino en comparación con $17 \%$ del sexo masculino, este hallazgo es similar a lo descrito por Toro y Cols. [6] quienes reportan una prevalencia de OMC en mujeres del $63.8 \%$ sometidas a timpanoplastía, discrepando por completo con Rodríguez [7] quien reporta una prevalencia del $54.8 \%$ en hombres y asemejándose a lo descrito por Waizel-Haiat y Cols. [8] quienes expresan que la OMC afecta con mayor frecuencia al sexo masculino. Así mismo, el sexo femenino se vio afectado a nivel del oído externo debido a otomicosis en un $65.7 \%$, respecto a esto, Escobar y Cols. [9] evidenciaron que la otomicosis fue más frecuente en mujeres, pese a esto, en la literatura médica existe discrepancia con respecto a este hallazgo, algunos autores como Hueso y Cols. [10] establecen una incidencia en varones del $55.8 \%$ lo que difiere un tanto de la obtenida por Fuentes y García [11] los cuales reportaron una prevalencia del $54 \%$ en hombres. La presbiacusia afecto en $12.8 \%$ 


\section{ASPECTOS CLINICOEPIDEMIOLÓGICOS DE LAS PATOLOGÍAS AMBULATORIAS DEL OIIDO FRECUENTES EN ADULTOS \\ PINEDA-GEA F}

al grupo poblacional evaluado y no se encontró distinción del sexo en la afectación de oído interno, lo que discrepa con Sousa y Cols. [12] quienes reportan una incidencia en hombres del $85.5 \%$ concordando su predomino del sexo masculino con Toledo y Cols. [13] los cuales mencionan una prevalencia de $69.5 \%$ discrepando con lo expresado por Ferre y Cols. [14] los cuales reportaron una prevalencia del $16.9 \%$ en el sexo femenino.

La principal comorbilidad identificada en pacientes con presbiacusia fue hipertensión arterial en el $30 \%$ de los casos, pese esto, se observó que el principal factor relacionado al desarrollo de esta entidad clínica fue en el $90 \%$ la edad $\geq$ de 51 años y no la presencia de esta comorbilidad, esto podría explicarse por el aumento en la sobrevida de la población o por una mejor cobertura y acceso a los servicios sanitarios, respecto a esto, Gutiérrez y Cols. [15] Demostraron que la hipertensión arterial se comporta tanto como causa de hipoacusia perceptiva o como factor contribuyente de presbiacusia, concluyendo que la hipertensión arterial es un factor que duplica la frecuencia e intensidad de esta, no obstante, Toledo y Cols [13] encontraron una asociación entre la presbiacusia y afecciones crónicas como las enfermedades cardiovasculares así mismo Ferre y Cols. [14] comprobaron una asociación estadística significativamente negativa en relación a la hipertensión arterial como factor etiológico asociado al riesgo de presbiacusia.

Tabla 6. Fármacos prescritos en las patologías ambulatorias prevalentes asociadas o no a otras patologías.

\begin{tabular}{|l|l|l|l|l|l|l|}
\hline $\begin{array}{l}\text { Fármacos } \\
\text { prescritos en } \\
\text { las patologías } \\
\begin{array}{l}\text { ambulatorias } \\
\text { prevalentes }\end{array}\end{array}$ & \multicolumn{2}{|l|}{$\begin{array}{l}\text { OMC } \\
(\mathrm{n}=189)\end{array}$} & \multicolumn{2}{|l|}{$\begin{array}{l}\text { Presbiacusia } \\
(\mathrm{n}=40)\end{array}$} & \multicolumn{2}{l|}{$\begin{array}{l}\text { Otomicosis } \\
(\mathrm{n}=38)\end{array}$} \\
\cline { 2 - 8 } & $\mathrm{n}$ & $\%$ & $\mathrm{n}$ & $\%$ & $\mathrm{n}$ & $\%$ \\
\hline $\begin{array}{l}\text { Ciprofloxacina } \\
500 \mathrm{mg} \text { PO }\end{array}$ & 109 & $\begin{array}{l}57.6 \\
7\end{array}$ & 7 & 17.5 & 20 & 52.6 \\
\hline
\end{tabular}

\begin{tabular}{|c|c|c|c|c|c|c|}
\hline \multirow{2}{*}{$\begin{array}{l}\text { Fármacos } \\
\text { prescritos en } \\
\text { las patologías } \\
\text { ambulatorias } \\
\text { prevalentes }\end{array}$} & \multicolumn{2}{|c|}{$\begin{array}{l}\text { OMC } \\
(n=189)\end{array}$} & \multicolumn{2}{|c|}{$\begin{array}{l}\text { Presbiacusia } \\
(\mathrm{n}=40)\end{array}$} & \multicolumn{2}{|c|}{$\begin{array}{l}\text { Otomicosis } \\
(\mathrm{n}=38)\end{array}$} \\
\hline & $\mathrm{n}$ & $\%$ & $\mathrm{n}$ & $\%$ & $\mathrm{n}$ & $\%$ \\
\hline $\begin{array}{l}\text { Ciprofloxacina } \\
500 \mathrm{mg} \text { PO, } \\
\text { ciprofloxacina } \\
0.2 \%+ \\
\text { dexametazona } \\
\text { ótica }\end{array}$ & 66 & 34.9 & 5 & 12.5 & 6 & 15.7 \\
\hline $\begin{array}{l}\text { Ciprofloxacina } \\
500 \mathrm{mg} \text { PO, } \\
\text { ciprofloxacina } \\
0.2 \%+ \\
\text { beclometazona } \\
\text { ótica }\end{array}$ & 18 & 9.5 & 1 & 2.5 & 1 & 2.6 \\
\hline $\begin{array}{l}\text { Ibuprofeno } \\
\text { 400mg PO }\end{array}$ & 26 & 13.7 & 1 & 2.5 & 6 & 15.7 \\
\hline $\begin{array}{l}\text { Fluconazol } \\
150 \mathrm{mg} \text { PO }\end{array}$ & 16 & 8.4 & 1 & 2.5 & 31 & 81.5 \\
\hline $\begin{array}{l}\text { Fluconazol } \\
\text { 150mg PO, } \\
\text { Clotrimazol } \\
\text { ótico }\end{array}$ & 9 & 4.7 & 0 & 0 & 16 & 42.1 \\
\hline $\begin{array}{l}\text { Fluconazol } \\
\text { 150mg PO, } \\
\text { beblometazona + } \\
\text { clioquinol ótico }\end{array}$ & 5 & 2.6 & 0 & 0 & 7 & 2.6 \\
\hline $\begin{array}{l}\text { Loratadina } \\
\text { 10mg PO }\end{array}$ & 173 & 91.5 & 18 & 45 & 28 & 73.6 \\
\hline $\begin{array}{l}\text { Dimenhidrinato } \\
50 \mathrm{mg} \text { PO }\end{array}$ & 1 & 0.5 & 3 & 7.5 & 2 & 5.2 \\
\hline $\begin{array}{l}\text { No se } \\
\text { prescribió } \\
\text { terapia } \\
\text { farmacológica }\end{array}$ & 6 & 3.1 & 19 & 47.5 & 1 & 0 \\
\hline
\end{tabular}

Encontramos que la otomicosis asociada a otitis media crónica prevaleció en 22 pacientes, los cuales recibieron tratamiento antibiótico por vía ótica por más de 10 días, el fluconazol junto con ciprofloxacino vía oral asociado a antifúngico tópico más corticoide, fue el tratamiento efectuado 


\section{ASPECTOS CLINICOEPIDEMIOLÓGICOS DE LAS PATOLOGÍAS AMBULATORIAS DEL OÍDO FRECUENTES EN ADULTOS \\ PINEDA-GEA F}

en estos pacientes, el uso de antimicóticos en la práctica clínica se alinea a lo expresado por la SEORL- CCC [16] la cual describe que el tratamiento de la otomicosis consiste en microaspiraciones y curas locales, junto con la aplicación de gotas anti-micóticas (Clotrimazol) 2-3 gotas cada 8 horas por 10-15 días. Así mismo Escobar y Cols.[9] mencionan que los azoles son los fármacos más eficaces contra la otomicosis y no suponen ningún tipo de ototoxicidad, esta descrito en la literatura, el hecho de que el tratamiento prolongado de gotas óticas tópicas con antibióticos, es una de las causas más frecuentes de otomicosis, por lo que basándonos en lo encontrado en nuestro estudio, expresamos que la prevalencia de la otomicosis en los pacientes con OMC, está en relación al uso prolongado de antibióticos por vía ótica u oral, cuya prescripción es respaldada por la reagudización del cuadro clínico, lo cual ocasiona, la salida de secreción a través del conducto auditivo externo produciendo un desequilibrio en la flora natural del mismo, favoreciendo el crecimiento de hongos, así mismo expresamos que la elección empírica del tratamiento antibiótico prescrito en los pacientes con patologías infecciosas concuerda con lo descrito por Mera y Cols. [17] Junto con Sabater y Cols. [18] Quienes mencionan que el ciprofloxacino es el fármaco sistémico y tópico elegido con mayor frecuencia en el tratamiento de afectaciones otológicas, debido a su acción frente a los gérmenes causantes de OMC reagudizada lo que justifica su uso en este grupo poblacional, ya que al revisar el expediente no se corroboró la toma, ni el reporte microbiológico confirmatorio del cultivo de secreción en estos pacientes. Ramos y Cols. [19] mencionan que la administración de ciprofloxacino por vía oral asociado a la administración por vía tópica no aportó beneficio alguno frente a las vías de administración exclusivamente tópicas, con respecto a esto Brennan y Cols. [20] expresan que existe mucha incertidumbre acerca de si los antibióticos tópicos mejoran o no la resolución de la secreción del oído en los pacientes con otitis media supurativa crónica. Pensamos que desde el punto de vista clínico, el abordaje terapéutico de las patologías ambulatorias del oído, no solo debe enfocarse al tratamiento sintomatológico sino que debe orientarse a obtener la curación completa del proceso causal, ya que como se observó en nuestro estudio la antibioterapia prolongada no es del todo eficaz para evitar las frecuentes recidivas y complicaciones, por lo que expresamos que el uso significativo de antibióticos orales, más allá de la terapia tópica, podría tener implicaciones en términos de costo, aparición de resistencia microbiana, riesgo de efectos secundarios y mayor probabilidad de incumplimiento terapéutico por parte del paciente, con respecto a esto Migirov y cols. [21] concluyen que el tratamiento con antibióticos no previene absolutamente el desarrollo de complicaciones intracraneales otogénicas, reportando una incidencia relativamente baja $(0.36 \%)$ concordando con nuestra investigación, en la cual pese al tratamiento antibiótico hubo un $0.96 \%$ que presento colesteatoma adquirido asociado a otomastoiditis crónica.

Tabla 7. Hallazgos clínicos presentados tras la terminación del tratamiento evidenciadas durante una segunda reevaluación de los pacientes.

\begin{tabular}{|c|c|c|c|c|c|c|}
\hline & \multicolumn{2}{|c|}{$\begin{array}{l}\text { OMC } \\
(\mathrm{n}=189)\end{array}$} & \multicolumn{2}{|c|}{$\begin{array}{l}\text { Presbiacusia } \\
(\mathrm{n}=40)\end{array}$} & \multicolumn{2}{|c|}{$\begin{array}{l}\text { Otomicosis } \\
(\mathrm{n}=38)\end{array}$} \\
\hline & $\mathrm{n}$ & $\%$ & $\mathrm{n}$ & $\%$ & $\mathrm{n}$ & $\%$ \\
\hline \multicolumn{7}{|c|}{ Hallazgos clínicos } \\
\hline $\begin{array}{l}\text { Disminución } \\
\text { de la capacidad } \\
\text { auditiva }\end{array}$ & 118 & 62.4 & 33 & 82.5 & 19 & 50 \\
\hline $\begin{array}{l}\text { Perforación } \\
\text { timpánica }\end{array}$ & 86 & 45.5 & 5 & 12.5 & 10 & 26.3 \\
\hline $\begin{array}{l}\text { Fracaso } \\
\text { terapéutico } \\
\text { por Infección } \\
\text { persistente del } \\
\text { oído medio y } \\
\text { externo }\end{array}$ & 27 & 14.1 & 2 & 5 & 1 & 2.6 \\
\hline $\begin{array}{l}\text { Acúfeno } \\
\text { persistente }\end{array}$ & 3 & 1.58 & 4 & 10 & 0 & 0 \\
\hline
\end{tabular}




\begin{tabular}{|l|l|l|l|l|l|l|}
\hline & \multicolumn{2}{|l|}{$\begin{array}{l}\text { OMC } \\
(\mathrm{n}=189)\end{array}$} & \multicolumn{2}{l|}{$\begin{array}{l}\text { Presbiacusia } \\
(\mathrm{n}=40)\end{array}$} & \multicolumn{2}{l|}{$\begin{array}{l}\text { Otomicosis } \\
(\mathrm{n}=38)\end{array}$} \\
\cline { 2 - 7 } & $\mathrm{n}$ & $\%$ & $\mathrm{n}$ & $\%$ & $\mathrm{n}$ & $\%$ \\
\hline Complicaciones clínicas \\
\hline Parálisis facial & 4 & 2.1 & 0 & 0 & 1 & 2.6 \\
\hline $\begin{array}{l}\text { Colesteatoma } \\
\text { adquirido } \\
\text { asociado a } \\
\text { otomastoiditis } \\
\text { crónica }\end{array}$ & 3 & 1.5 & 0 & 0 & 0 & 0 \\
\hline Ninguna & 38 & 20.1 & 5 & 12.5 & 18 & 47.3 \\
\hline
\end{tabular}

Las comorbilidades identificadas en el grupo poblacional que presento OMC, presbiacusia y otomicosis, fueron diabetes mellitus tipo 2 e hipertensión arterial, cabe recalcar que en estos pacientes, hubo un porcentaje del $7.05 \%$ que presento simultáneamente la combinación de estas dos comorbilidades. Lo que podría asociarse al hecho que el aumento de la prevalencia de estas, se deba al aumento de la población, su envejecimiento y a factores de riesgo relacionados con una dieta malsana, inactividad física, sobrepeso, entre otros. En lo que respecta a los hallazgos clínicos encontrados tras una segunda reevaluación, se evidencio que la hipoacusia fue el principal síntoma referido post tratamiento, por lo que expresamos que esto quizá este en relación con la resolución del cuadro clínico, así mismo destacamos que el $82.5 \%$ de los pacientes con presbiacusia refirió este síntoma, cabe mencionar que pese a que en estos pacientes hubo una resolución satisfactoria del cuadro infeccioso asociado, se les indico además, el uso de auxiliar auditivo en la consulta previa y ninguno de estos pacientes refirió haber consultado un audiólogo protésico, esto podría explicarse por el hecho que en nuestro medio existe una limitada accesibilidad a este tipo de servicio profesional. La principal complicación clínica evidenciada fue parálisis facial en el $1.5 \%$ de los pacientes con OMC, los cuales presentaron una o más reagudizaciones, datos similares expresan Bordure y Cols. [22] Quienes estiman su frecuencia en el 1-2 \% en pacientes con OMC asociada a colesteatoma.
Las manifestaciones clínicas referidas al momento de la consulta en pacientes con OMC fueron: hipoacusia, otalgia, otorrea, los últimos dos síntomas concuerdan con la distribución de síntomas expresados por Cremé-Aguirre y Cols. [23] los cuales mencionan que la otalgia es un síntoma muy raro en otitis media crónica y su presentación clínica debe sugerir una reagudización, hecho que fundamenta en nuestro estudio la presencia de este síntoma con mayor predomino en pacientes con procesos infecciosos activos, por su parte Rodríguez [7] detalla que la otorrea es el síntoma fundamental en la OMC, esta descrito que la hipoacusia de tipo conductiva es la más frecuente en OMC y usualmente su persistencia se asocia a ocupación de la caja timpánica por fluidos que no se eliminan. Cabe mencionar que estas manifestaciones clínicas no fueron referidas de manera exclusiva por la población, sino que se presentaron de manera combinada concordando con Mera y Cols. [17] y Guranga y Arguello [24] Los cuales describen que estos síntomas pueden asociarse a procesos agudos o crónicos. En cuanto a la presencia de prurito ótico en otomicosis Ruz y Cols. [4] lo consideran como un síntoma patognomónico que puede ser referido de manera exclusiva o asociado a otros síntomas, tal como se evidenció en esta investigación.

Es importante destacar que en nuestro estudio pese a que la hipoacusia fue el principal signo y síntoma clínico evidenciado en la anamnesis y reporte audiométrico, no se recogió la información sobre el perfil audiológico presentado por el paciente, sin embargo se registró la presencia de estas pruebas en el expediente clínico, por lo que constatamos que en el $100 \%(n=312)$ de los pacientes se les realizó un estudio otorrinolaringológico que incluía una exploración otomicroscópica, audiométrica, timpanometríca Desde el punto de vista imagenológico, la TAC cráneo y oído simple (con cortes a $1 \mathrm{~mm}$ ), se utilizó en pacientes con OMC con antecedentes de más de dos o tres reagudizaciones, con el objetivo de descartar ocupación 


\section{ASPECTOS CLINICOEPIDEMIOLÓGICOS DE LAS PATOLOGÍAS AMBULATORIAS DEL OÍDO FRECUENTES EN ADULTOS \\ PINEDA-GEA F}

de celdillas mastoideas, lesiones osteolíticas en oído medio o colestomatosas. La resonancia magnética fue realizada a un paciente que presento diagnóstico de shwanoma vestibular de oído izquierdo el cual tuvo que ser reintervenido por infección de sitio quirúrgico, así mismo fue el único paciente al que se realizó cultivo de secreción reportándose infección por Acinetobacter baumannii. Expresamos que estas técnicas de imagen diagnósticas, fueron utilizadas en nuestro estudio con el objetivo de excluir o delimitar las lesiones estructurales del oído en pacientes con antecedentes infecciosos a repetición; Cabe mencionar que no a todos los pacientes con OMC se realizó estas técnicas, ya que su utilización, esta mediada por el cumplimiento de ciertos criterios clínicos asociados con la gravedad del proceso, el estado general del paciente, comorbilidades coexistentes, respuesta clínica ante la terapia farmacológica entre otros, los cuales suponen factores que pesan en contra de la indicación de estas estas técnicas diagnósticas, ya que en la medida de lo posible en la práctica clínica, se pretende minimizar no solo la exposición de estos pacientes a altas dosis de radiación, sino también el costo que genera la indicación innecesaria de estas técnicas.

El fármaco prescrito con mayor frecuencia en OMC, presbiacusia y otomicosis, fue loratadina, sin en cambio, llama la atención su prescripción en la mayoría de los pacientes diagnosticados con OMC sin proceso infeccioso activo, agregando a esto el hecho que no se encontró descrito en el expediente clínico alguna afecciones de órganos adyacentes: estados alérgicos nasales, infecciones rinosinusales y faríngeas; que pudieran influir en el curso de la enfermedad y que justificará la prescripción de este fármaco en estos estos pacientes, añadiendo que no se encontró en la literatura médica estudios que avalaran esta práctica clínica; con respecto a esto Griffin y Flynn [25] expresan que no hay ningún beneficio o algún daño por el uso de antihistamínicos o descongestionantes, solos o en combinación en el manejo de la otitis media crónica, por lo tanto, recomiendan no usarlos, lo que establece la duda del porqué de su prescripción, evidenciando una insuficiente fundamentación diagnóstica y terapéutica, por lo que su indicación en estos pacientes es cuestionable. En base a lo anterior, expresamos que el prescriptor quizá sea vulnerable a influencias que puedan causar una prescripción irracional como lo es la presión de los familiares o el mismo paciente, que en su desesperación de cambiar el curso natural de la enfermedad, orilla al profesional de la salud a tomar la decisión de prescribir este fármaco como efecto placebo.

\section{CONCLUSIONES}

En base a los resultados obtenidos, bajo las condiciones del estudio, se puede concluir que según el diagnóstico, las enfermedades más frecuentes que motivaron la consulta correspondieron a otitis media crónica, presbiacusia y otomicosis las cuales tuvieron una mayor prevalencia en el sexo femenino, Discrepando con lo reportado en otras investigaciones, se manifestaron con hipoacusia otalgia, otorrea y prurito ótico El diagnóstico se alineó a lo descrito por la literatura el cual se realizó mediante pruebas audiológicas (Otoscopia, audiometría y timpanometría) e imagenológicas (TAC de cráneo y oído simple), estas últimas se reservó en pacientes con OMC reagudizada, con el fin de descartar o delimitar el daño estructural del oído. Desde el punto de vista terapéutico todos los casos se trataron de manera empírica, encontrándose que la prescripción de antihistamínicos (Loratadina) en estos pacientes fue común en la práctica clínica pese a la escasa fundamentación terapéutica. Así mismo se identificó que el uso significativo de antibióticos orales, más allá de la terapia tópica, no es del todo eficaz para evitar recidivas, lo que podría tener implicaciones en términos de costo, aparición de resistencia microbiana, riesgo de efectos secundarios y mayor probabilidad de incumplimiento terapéutico por parte del paciente. 


\section{ASPECTOS CLINICOEPIDEMIOLÓGICOS DE LAS PATOLOGÍAS AMBULATORIAS DEL OÍDO FRECUENTES EN ADULTOS \\ PINEDA-GEA F}

\section{DECLARACIÓN DE INTERESES}

El estudio forma parte del trabajo de titulación de médico y cirujano general presentado por la autora en la universidad Católica Redemptoris Mater (UNICA), El 27 de junio del 2019.

\section{AGRADECIMIENTOS}

Agradezco a Francisca Gea López, Arturo y Sofía Pineda Gea, Marco Bolaños, quienes fueron parte medular de mi formación, de manera especial al personal del departamento de estadística del hospital escuela Antonio Lenín Fonseca quienes contribuyeron de forma excepcional en esta investigación proporcionándome el acceso a los expedientes clínicos así mismo agradezco por su valiosa colaboración en esta investigación al Dr. José Luis Pardal Refoyo junto con el equipo de edición de esta revista, por su paciencia y dedicación para guiarme en la edición de este artículo.

\section{BIBLIOGRAFÍA}

1. Seymour M Camila, Pardo J Javiera, Bahamonde $S$ Héctor. Otorrinolaringología patología ambulatoria en el Hospital Clínico de la Universidad de Chile en 2008: Informe de 9.157 pacientes. Rev. Otorrinolaringol. Cir. Cabeza Cuello.2011, Dic; 71 (3): 237-240. Disponible en: http://dx.doi. org/10.4067/S0718-48162011000300008 [Citado el 07/05/2020]

2. Castaño Z., R. Otitis media crónica como problema de salud pública en países en vía de desarrollo Rev. Colombina de Otorrinolaringología. 2016. Nov; 29(3) Disponible en: https://encolombia. com/medicina/revistas-medicas/aoccc/vol-293/ otorrino29301a-otitis/ [Citado el 07/05/2020]

3. Piura López J. Metodología de la investigación científica: Un enfoque integrador. $7 \mathrm{ma}$ ed. Managua, Nicaragua: Editorial PAVSA. 2012. pp. 28-223

4. Ruz G S, Breinbauer K H, Arancibia SM. Análisis epidemiológico de la patología otorrinolaringológica ambulatoria en el Hospital San Juan de Dios. Rev. Otorrinolaringol. Cir. Cabeza Cuello 2009.
Dic; 69(3):227-232. Disponible en: http://dx.doi. org/10.4067/S0718-48162009000300004 [Citado el 30/04/2020]

5. Browning GG, Gatehouse S. La prevalencia de la enfermedad del oído medio en la población adulta británica. Otorrinolaringología clínica y ciencias afines. 1992. Agost; 17 (4): 317-321. Disponible en: https://doi.org/10.1111/j.1365-2273.1992. tb01004.x [Citado el 07/05/2020]

6. Toro A C, Naser G A, Sanhueza L C, Valdés P C, Gormaz B J P, Molina C V et al. Timpanoplastía en pacientes adultos en el Hospital Clínico de la Universidad de Chile: revisión de la experiencia de 10 años. Rev. Otorrinolaringol. Cir. Cabeza Cuello 2007 Dic 67 (3): 237-243. Disponible en: http:// dx.doi.org/10.4067/S0718-48162007000300005 [Citado el 24/03/2020]

7. Rodríguez Panadés Y. Características clínicoepidemiológicas y correspondencia video- otoscópico-audiológica de la otitis media crónica simple. Rev Cubana Otorrinolaringol Cirug Cabeza Cuello 2019 3(1): 1. Disponible en: http://revotorrino. sld.cu/index.php/otl/article/view/7 2 [Citado el 24/03/2020]

8. Waizel Haiat S, Copado Ceballos R E, Rojas Barrera L, Vargas Aguayo AM. Absceso de Luc en el siglo XXI. Rev Mex AMCAOF. 2012; 1 (2): 104-112 Disponible en: https://www.researchgate.net/profile/Salomon_W aizelHaiat/ publication/281210029_Absceso_de_Luc_en el_siglo_XXI/links/55db936308aec156b 9afee49. pdf [Citado el 24/02/2019]

9. Escobar Lucero, Celis Erika, Alarid José, Jiménez Lesly, Díaz Gaudencio, Muñoz Víctor. studio clínico y micológico de otomicosis en diabéticos: Una serie de 17 casos. Rev. Otorrinolaringol. Cir. Cabeza Cuello 2018; 78: 36-42 Disponible en: https://www.researchgate.net/publication/324579 784_Estudio_clinico_y_micologico_de_otomicosi s_en_diabeticos_Una_serie_de_17_casos [Citado el 24/03/2020]

10. Hueso Gutiérrez P., Jiménez Álvarez S, GilCarcedo Sañudo E, Gil-Carcedo García L.M, Ramos Sánchez C, \& Vallejo Valdezate L A. Diagnóstico de presunción: Otomicosis. Estudio de 451 pacientes. Rev. Acta Otorrinolaringo- 


\section{ASPECTOS CLINICOEPIDEMIOLÓGICOS DE LAS PATOLOGÍAS AMBULATORIAS DEL OÍDO FRECUENTES EN ADULTOS \\ PINEDA-GEA F}

lógica Española, 2005; Abril 56(5), 181-186. Disponible en: https://doi.org/10.1016/S00016519(05)78597-6 [Citado el 14/04/2020]

11. Fuentes Anaya MR, García-Calderón-Sandoval M. Otomicosis: Prevalencia, etiología y terapéutica en el Servicio de Otorrinolaringología del Hospital Nacional Hipólito Unanue. Sociedad Peruana de Otorrinolaringología y Cirugía Facial.2005,Jun;(1), 15-16 Disponible en: http:// repebis.upch.edu.pe/articulos/reporl/v29n1/ a2.pdf [Citado el 14/04/2020]

12. Sousa Cláudia Simônica de, Castro Júnior Ney de, Larsson Erkki Juhani, Ching Ting Hui. Estudo de fatores de risco para presbiacusia em indivíduos de classe sócio-econômica média. Braz. j. otorhinolaryngol. (Impr.) 2009 Aug; 75(4): 530-536. Disponible en: https://doi.org/10.1590/ S1808- 86942009000400011 [cited 2020 Mar 24]

13. Toledo Valdés C, Pacheco Macías AR, Pérez García T, Contreras Álvarez PJ, Hernández Armstrong L. Características clínico- epidemiológicas de pacientes ancianos con Hipoacusia atendidos en el Hospital Calixto García. Revista Habanera de Ciencias Médicas. 2018; 17(3): 12. Disponible en: http://www.revhabanera.sld.cu/ index.php/rhab/art icle/view/2152 [Citado el 10/05/2020]

14. Ferré rey J, Morelló-castro G, \& Barberá curto JL. (2002). Factores de riesgo involucrados en la presbiacusia. Acta Otorrinolaringológica Española, 2002, Mayo; 53(8):572-577. Disponible en: https://doi.org/10.1016/S0001- 6519(02)78350-7 [Citado el 30/04/2020]

15. Gutiérrez Baryolo E, Ferrer Herrera I, Ferrer Murgas G, Ferrer Tan I. Repercusión de la hipertensión arterial sobre la audición. Rev Arch Méd Camagüey 2015; 1(2): [aprox. 0 p.]. Disponible en: http://www.revistaamc.sld.cu/index.php/ amc/articl e/view/3659 [Citado el 24/03/2020]

16. Sociedad Española de Otorrinolaringología y Patología Cérvico-Facial (2014). Libro virtual de formación en otorrinolaringología 1 Ed. capítulos 13-35. Disponible en. https://es.scribd. com/document/359826669/Libro-Virtual-deFormacion-en-Otorrinolaringologia- SEORL [Citado el 24/05/2019]
17. Mera-Menéndez F, Álvarez-Carrillo S, LópezRamos D. Otorrea. FMC Curso 2008; 15 (Extraordin 1):5-9. Disponible en: http://www. sspa.juntadeandalucia.es/servicioandaluzdesalud/hinmaculada/intranet/ugcolula/guias/ORL/ OTORREA.pdf Citado el 24/06/2019]

18. Sabater Mata F, Pumarola F, Ballesteros F, Homs I. ¿Está indicado el tratamiento tópico en las otitis que cursan con otorrea en el niño? Aportación terapéutica de ciprofloxacina tópico. REV ESP PEDIATR 2008. 64(3):193-199 Disponible en: https://www.seinap.es/wp-content/uploads/ Revista-de-Pediatria/2008/REP\%2064-3. pdf\#page=5 [Citado el 24/02/2019].

19. Ramos A, Ayudarte F, De Miguel I, Cuyás J.M, \& Cenjor, C. Utilización del ciprofloxacino tópico en la otitis media crónica supurada. Acta Otorrinolaringológica Española, 2003; 54(7), 485-490. Disponible en: https://doi.org/10.1016/S00016519(03)78439-8 [Citado el 30/03/2020]

20. Brennan-Jones CG, Head K, Chong LY, Burton MJ, Schilder AGM, Bhutta MF. Topical antibiotics for chronic suppurative otitis media. Cochrane Database of Systematic Reviews 2020, Issue 1. Art. No.: CD013051. Disponible en: https://doi. org/10.1002/14651858.CD013051.pub 2 [Citado el 24/03/2020]

21. Migirov L, Duvdevani S, Kronenberg J. Complicaciones intracraneales otogénicas: una revisión de 28 casos. Acta Otolaryngol 2005; 125(8): 819. Disponible en: https://www.uptodate. com/contents/acute-otitis- media-in-adults/ abstract/53 [Citado el 10/05/2020]

22. Bordure, P., Bailleul, S., Malard, O. y Wagner, R. (2010). Otitis crónica colesteatomatosa. Aspectos clínicos y terapéuticos. EMC - Otorrinolaringología, 39 (1), 1-16. Disponible en: https://doi. org/10.1016/S1632-3475(10)70275-7 [Citado el 7/05/2020]

23. Cremé-Aguirre J. Bueno-González E. Sevilla-Salas M. Figueras Hechavarria I. Romero-Formeta R. Chacón-Benítez O. Rosales-Sánchez M. (2006). Caracterización de ancianos en consulta externa de otorrinolaringología. Guantánamo, Cuba. Revista Información Científica 2006, 51 (3) Disponible en: https://www.redalyc.org/pdf/5517/55175733 4001.pdf [Citado el 24/03/2019] 


\section{ASPECTOS CLINICOEPIDEMIOLÓGICOS DE LAS PATOLOGÍAS AMBULATORIAS \\ DEL OÍDO FRECUENTES EN ADULTOS \\ PINEDA-GEA F}

24. Guaranga-Gualli F W, Argüello F. Factores de riesgo y complicaciones de la otitis media crónicas en edades de 18 a 40 años en el hospital León Becerra de Milagro durante el periodo 2013 a 2015. Guayaquil-Ecuador. Repositorio institucional de la universidad de Guayaquil. 2016. Disponible en: http://repositorio.ug.edu. ec/bitstream/redug/23234/1/CD\%201123-\%20
GUARANGA\%20GUALLI\%20FRANKLIN\%20 WASHINGTON.pdf [Citado el 24/02/2019]

25. Griffin G, Flynn CA. Antihistamines and/or decongestants for otitis media with effusion (OME) in children. Cochrane Database of Systematic Reviews 2011, Issue 9. Art. No.: CD003423. Disponible en: https://doi.org/10.1002/14651858. CD003423.pub 3. [Citado el 6/05/2020] 